\title{
Moudafi's open question and simultaneous iterative algorithm for general split equality variational inclusion problems and general split equality optimization problems
}

Shih-sen Chang ${ }^{*}$, Lin Wang, Yong Kun Tang and Gang Wang

*Correspondence:

changss2013@163.com College of Statistics and

Mathematics, Yunnan University of Finance and Economics, Kunming, Yunnan 650221, P.R. China

\begin{abstract}
The purpose of this paper is first to introduce and study the general split equality variational inclusion problems and the general split equality optimization problems in the setting of infinite-dimensional Hilbert spaces and then propose a new simultaneous iterative algorithm. Under suitable conditions, some strong convergence theorems for the sequences generated by the proposed algorithm converging strongly to a solution for these two kinds of problems are proved. As special cases, we shall utilize our results to study the split feasibility problems, the split equality equilibrium problems, and the split optimization problems. The results presented in the paper not only extend and improve the corresponding recent results announced by many authors, but they also provide an affirmative answer to an open question raised by Moudafi in his recent work.
\end{abstract}

Keywords: general split equality variational inclusion problem; general split equality optimization problem; split feasibility problem; split equality equilibrium problem; split optimization problem

\section{Introduction}

Let $C$ and $Q$ be nonempty closed convex subsets of real Hilbert spaces $H_{1}$ and $H_{2}$, respectively. The split feasibility problem (SFP) is formulated as

$$
\text { to finding } x^{*} \in C \text { and } A x^{*} \in Q \text {, }
$$

where $A: H_{1} \rightarrow H_{2}$ is a bounded linear operator. In 1994, Censor and Elfving [1] first introduced the (SFP) in finite-dimensional Hilbert spaces for modeling inverse problems which arise from phase retrievals and in medical image reconstruction [2]. It has been found that the (SFP) can also be used in various disciplines such as image restoration, and computer tomograph and radiation therapy treatment planning [3-5]. The (SFP) in an infinite-dimensional real Hilbert space can be found in [2, 4, 6-10].

Assuming that the (SFP) is consistent, it is not hard to see that $x^{*} \in C$ solves (SFP) if and only if it solves the fixed-point equation

$$
x^{*}=P_{C}\left(I-\gamma A^{*}\left(I-P_{Q}\right) A\right) x^{*},
$$

@2014 Chang et al.; licensee Springer. This is an Open Access article distributed under the terms of the Creative Commons Attribution License (http://creativecommons.org/licenses/by/2.0), which permits unrestricted use, distribution, and reproduction in any medium, provided the original work is properly cited. 
where $P_{C}$ and $P_{Q}$ are the metric projection from $H_{1}$ onto $C$ and from $H_{2}$ onto $Q$, respectively, $\gamma>0$ is a positive constant and $A^{*}$ is the adjoint of $A$.

A popular algorithm to be used to solves the SFP (1.1) is due to Byrne's CQ-algorithm [2]:

$$
x_{k+1}=P_{C}\left(I-\gamma A^{*}\left(I-P_{Q}\right) A\right) x_{k}, \quad k \geq 1,
$$

where $\gamma \in(0,2 / \lambda)$ with $\lambda$ being the spectral radius of the operator $A^{*} A$.

Recently, Moudafi $[11,12]$ introduced the following split equality feasibility problem (SEFP):

$$
\text { to find } x \in C, y \in Q \text { such that } A x=B y \text {, }
$$

where $A: H_{1} \rightarrow H_{3}$ and $B: H_{2} \rightarrow H_{3}$ are two bounded linear operators. Obviously, if $B=I$ (identity mapping on $\mathrm{H}_{2}$ ) and $H_{3}=H_{2}$, then (1.3) reduces to (1.1). The kind of split equality problems (1.3) allows asymmetric and partial relations between the variables $x$ and $y$. The interest is to cover many situations, such as decomposition methods for PDEs, and applications in game theory and intensity-modulated radiation therapy.

In order to solve the split equality feasibility problem (1.3), Moudafi [11] introduced the following simultaneous iterative method:

$$
\left\{\begin{array}{l}
x_{k+1}=P_{C}\left(x_{k}-\gamma A^{*}\left(A x_{k}-B y_{k}\right)\right), \\
y_{k+1}=P_{Q}\left(y_{k}+\beta B^{*}\left(A x_{k+1}-B y_{k}\right)\right),
\end{array}\right.
$$

and under suitable conditions he proved the weak convergence of the sequence $\left\{\left(x_{n}, y_{n}\right)\right\}$ to a solution of (1.3) in Hilbert spaces.

At the same time, he raised the following open question.

Moudafi's Open Question 1.1 Is there any strong convergence theorem of an alternating algorithm for the split equality feasibility problem (1.3) in real Hilbert spaces?

More recently, Eslamian and Latif [13], Chen et al. [14], Chuang [15] and Chang and Wang [16] introduced and studied some kinds of general split feasibility problem, general split equality problem, and split variational inclusion problem in real Hilbert spaces. Under suitable conditions some strong convergence theorems are proved. Also a comprehensive survey and update bibliography on split feasibility problems are given in Ansari and Rehan [17].

Motivated by the above works and related literature, in this paper, we continue to consider the problem (1.3). We obtain some strongly convergent theorems to a solution of the problem (1.3) which provide an affirmative answer to Moudafi's open question.

For the purpose we first introduce and consider the following more general problems.

(I) General split equality variational inclusion problem:

(GSEVIP) to find $x^{*} \in H_{1}$ and $y^{*} \in H_{2}$ such that

$$
0 \in \bigcap_{i=1}^{\infty} U_{i}\left(x^{*}\right), \quad 0 \in \bigcap_{i=1}^{\infty} K_{i}\left(y^{*}\right) \text { and } A x^{*}=B y^{*},
$$


where $H_{1}, H_{2}$ and $H_{3}$ are three real Hilbert spaces, $U_{i}: H_{1} \rightarrow H_{1}$ and $K_{i}: H_{2} \rightarrow H_{2}, i=$ $1,2, \ldots$ are two families of set-valued maximal monotone mappings, $A: H_{1} \rightarrow H_{3}$ and $B$ : $\mathrm{H}_{2} \rightarrow \mathrm{H}_{3}$ are two linear and bounded operators.

(II) General split equality optimization problem:

(GSEOP) to find $x^{*} \in H_{1}$ and $y^{*} \in H_{2}$ such that for each $i \geq 1$

$$
h_{i}\left(x^{*}\right)=\min _{x \in H_{1}} h_{i}(x), \quad g_{i}\left(y^{*}\right)=\min _{y \in H_{2}} g_{i}(y) \quad \text { and } \quad A x^{*}=B y^{*},
$$

where $H_{1}, H_{2}$, and $H_{3}$ are three real Hilbert spaces, $A: H_{1} \rightarrow H_{3}$ and $B: H_{2} \rightarrow H_{3}$ are two linear and bounded operators, $h_{i}: H_{1} \rightarrow \mathbb{R}$ and $g_{i}: H_{2} \rightarrow \mathbb{R}$ are two countable families of proper, convex, and lower semicontinuous functions.

The following problems are special cases of Problem I and II.

(III) Split equality feasibility problems.

Let $C \subset H_{1}$ and $Q \subset H_{2}$ be two nonempty closed convex subsets and $A: H_{1} \rightarrow H_{3}$, $B: H_{2} \rightarrow H_{3}$ be two bounded linear operators. As mentioned above the so-called 'split equality feasibility problem' (SEFP) is to find

$$
x^{*} \in C, y^{*} \in Q \text { such that } A x^{*}=B y^{*} \text {. }
$$

Let $i_{C}$ and $i_{Q}$ be the indicator functions of $C$ and $Q$, respectively, i.e.,

$$
i_{C}(x)=\left\{\begin{array}{ll}
0, & \text { if } x \in C, \\
+\infty, & \text { if } x \notin C ;
\end{array} \quad i_{Q}(y)= \begin{cases}0, & \text { if } y \in C \\
+\infty, & \text { if } y \notin Q\end{cases}\right.
$$

Denote by $N_{C}(x)$ and $N_{Q}(y)$ the normal cones of $C$ and $Q$ at $x$ and $y$, respectively:

$$
\begin{aligned}
& N_{C}(x)=\left\{z \in H_{1}:\langle z, v-x\rangle \leq 0, \forall v \in C\right\}, \\
& N_{Q}(y)=\left\{z \in H_{2}:\langle z, v-y\rangle \leq 0, \forall v \in Q\right\} .
\end{aligned}
$$

It is easy to know that $i_{C}$ and $i_{Q}$ both are proper convex and lower semicontinuous functions on $H_{1}$ and $H_{2}$, respectively, and the sub-differentials $\partial i_{C}$ and $\partial i_{Q}$ both are maximal monotone operators. We define the resolvent operator $J_{\beta}^{\partial i_{C}}$ of $i_{C}$ by

$$
J_{\beta}^{\partial i_{C}}(x)=\left(I+\beta \partial i_{C}\right)^{-1}(x), \quad \beta>0, x \in H_{1} .
$$

Here

$$
\begin{aligned}
\partial i_{C}(x) & =\left\{z \in H_{1}: i_{C}(x)+\langle z, u-x\rangle \leq i_{C}(u), \forall u \in H_{1}\right\} \\
& =\left\{z \in H_{1}:\langle z, u-x\rangle \leq 0, \forall u \in C\right\}=N_{C}(x), \quad x \in C .
\end{aligned}
$$

Hence we have

$$
\begin{aligned}
u & =J_{\beta}^{\partial i_{C}}(x) \quad \Leftrightarrow \quad x-u \in \beta N_{C}(u) \\
& \Leftrightarrow \quad\langle x-u, y-u\rangle \leq 0, \quad \forall y \in C \quad \Leftrightarrow \quad u=P_{C}(x) .
\end{aligned}
$$

This implies that $J_{\beta}^{\partial i_{C}}=P_{C}$ for any $\beta>0$. Similarly, we also have $\partial i_{Q}(y)=N_{Q}(y)$, and $J_{\beta}^{\partial i_{Q}}=$ $P_{Q}$ for any $\beta>0$. Therefore the (SEFP) (1.3) is equivalent to the following split equality 
optimization problem, i.e., to find $x^{*} \in H_{1}$, and $y^{*} \in H_{2}$ such that

$$
\begin{gathered}
i_{C}\left(x^{*}\right)=\min _{x \in H_{1}} i_{C}(x), \quad i_{Q}\left(y^{*}\right)=\min _{y \in H_{2}} i_{Q}(y) \quad \text { and } A x^{*}=B y^{*} ; \\
\Leftrightarrow \quad 0 \in \partial i_{C}\left(x^{*}\right), \quad 0 \in \partial i_{Q}\left(y^{*}\right) \quad \text { and } A x^{*}=B y^{*} .
\end{gathered}
$$

(IV) Split equality equilibrium problem.

Let $D$ be a nonempty closed and convex subset of a real Hilbert space $H$. A bifunction $g: D \times D \rightarrow(-\infty,+\infty)$ is said to be a equilibrium function, if it satisfies the following conditions:

(A1) $g(x, x)=0$, for all $x \in D$;

(A2) $g$ is monotone, i.e., $g(x, y)+g(y, x) \leq 0$ for all $x, y \in D$;

(A3) $\lim \sup _{t \downarrow 0} g(t z+(1-t) x, y) \leq g(x, y)$ for all $x, y, z \in D$;

(A4) for each $x \in D, y \mapsto g(x, y)$ is convex and lower semicontinuous.

The so-called equilibrium problem with respect to the equilibrium function $g$ is

to find $x^{*} \in D$ such that $g\left(x^{*}, y\right) \geq 0, \quad \forall y \in D$.

Its solution set is denoted by $E P(g)$.

For given $\lambda>0$ and $x \in H$, the resolvent of the equilibrium function $g$ is the operator $R_{\lambda, g}: H \rightarrow D$ defined by

$$
R_{\lambda, g}(x):=\left\{z \in D: g(z, y)+\frac{1}{\lambda}\langle y-z, z-x\rangle \geq 0, \forall y \in D\right\}
$$

Proposition 1.2 [18] The resolvent operator $R_{\lambda, g}$ of the equilibrium function $g$ has the following properties:

(1) $R_{\lambda, g}$ is single-valued;

(2) $F\left(R_{\lambda, g}\right)=E P(g)$ and $E P(g)$ is a nonempty closed and convex subset of $D$;

(3) $R_{\lambda, g}$ is a firmly nonexpansive mapping.

Let $h, g: D \times D \rightarrow(-\infty,+\infty)$ be two equilibrium functions. For given $\lambda>0$, let $R_{\lambda, h}$ and $R_{\lambda, g}$ be the resolvent of $h$ and $g$ (defined by (1.9)), respectively.

The so-called split equality equilibrium problem with respective to $h, g$, and $D$ $(\operatorname{SEEP}(h, g, D))$ is to find $x^{*} \in D, y^{*} \in D$ such that

$$
h\left(x^{*}, u\right) \geq 0, \quad \forall u \in D, \quad g\left(y^{*}, v\right) \geq 0, \quad \forall v \in D \quad \text { and } \quad A x^{*}=B y^{*}
$$

where $A, B: D \rightarrow D$ are two linear and bounded operators.

By Proposition 1.2, the $(\operatorname{SEEP}(h, g, D))(1.10)$ is equivalent to find $x^{*} \in D, y^{*} \in D$ such that for each $\lambda>0$

$$
\begin{aligned}
& x^{*} \in E P(h, D), \quad y^{*} \in E P(g, D) \quad \text { and } A x^{*}=B y^{*} \\
& \quad \Leftrightarrow \quad x^{*} \in F\left(R_{\lambda h}\right), \quad y^{*} \in F\left(R_{\lambda g}\right) \text { and } A x^{*}=B y^{*} .
\end{aligned}
$$

Letting $C=F\left(R_{\lambda h}\right), Q=F\left(R_{\lambda g}\right)$, by Proposition 1.2, $C$ and $Q$ both are nonempty closed and convex subset of $D$. Hence the problem (1.10) is equivalent to the following split equality 
feasibility problem:

$$
\text { to find } x^{*} \in C, y^{*} \in Q \text { such that } A x^{*}=B y^{*} \text {. }
$$

\section{(V) Split optimization problem.}

Let $H_{1}$ and $H_{2}$ be two real Hilbert spaces, $A: H_{1} \rightarrow H_{2}$ be a linear and bounded operators, $h: H_{1} \rightarrow \mathbb{R}$ and $g: H_{2} \rightarrow \mathbb{R}$ be two proper convex and lower semicontinuous functions. The split optimization problem (SOP) is to find $x^{*} \in H_{1}, A x^{*} \in H_{2}$ such that

$$
h\left(x^{*}\right)=\min _{x \in H_{1}} h_{i}(x) \text { and } g\left(A x^{*}\right)=\min _{z \in H_{2}} g(z) .
$$

Denote by $U=\partial h$ and $K=\partial g$, then the (SOP) (1.12) is equivalent to the following split variational inclusion problem (SVIP): to find $x^{*} \in H_{1}$ such that

$$
0 \in U\left(x^{*}\right), \quad 0 \in K\left(A x^{*}\right) .
$$

For solving (GSEVIP) (1.5) and (GSEOP) (1.6), in Sections 3 and 4, we propose a new simultaneous type iterative algorithm. Under suitable conditions some strong convergence theorems for the sequences generated by the algorithm are proved in the setting of infinitedimensional Hilbert spaces. As special cases, we shall utilize our results to study the split feasibility problem, split equality equilibrium problem and the split optimization problem. By the way, we obtain a strongly convergent iterative sequence to a solution of the problem (1.3), which provides an affirmative answer to the open question raised by Moudafi [11]. The results presented in the paper extend and improve the corresponding results announced by Moudafi et al. [11, 12, 19], Eslamian and Latif [13], Chen et al. [14], Censor et al. [1, 3-5, 20], Chuang [15], Naraghirad [21], Chang and Wang [16], Ansari and Rehan [17], and some others.

\section{Preliminaries}

We first recall some definitions, notations, and conclusions.

Throughout this paper, we assume that $H$ is a real Hilbert space and $C$ is a nonempty closed convex subset of $H$. In the sequel, we denote by $F(T)$ the set of fixed points of a mapping $T$ and by $x_{n} \rightarrow x^{*}$ and $x_{n} \rightarrow x^{*}$, the strong convergence, and weak convergence of a sequence $\left\{x_{n}\right\}$ to a point $x^{*}$, respectively.

Recall that a mapping $T: H \rightarrow H$ is said to be nonexpansive, if $\|T x-T y\| \leq\|x-y\|$, $\forall x, y \in H$. A typical example of nonexpansive mapping is the metric projection $P_{C}$ from $H$ onto $C \subseteq H$ defined by $\left\|x-P_{C} x\right\|=\inf _{y \in C}\|x-y\|$. The metric projection $P_{C}$ is firmly nonexpansive, if

$$
\left\|P_{C} x-P_{C} y\right\|^{2} \leq\left\langle x-y, P_{C} x-P_{C} y\right\rangle \quad \forall x, y \in H,
$$

and it can be characterized by the fact that

$$
P_{C}(x) \in C \quad \text { and } \quad\left\langle y-P_{C}(x), x-P_{C}(x)\right\rangle \leq 0, \quad \forall x \in H, y \in C .
$$

A mapping $T: H \rightarrow H$ is said to be quasinonexpansive, if $F(T) \neq \emptyset$, and

$$
\|T x-p\| \leq\|x-p\|, \quad \text { for each } x \in H \text { and } p \in F(T) .
$$


It is easy to see that if $T$ is a quasi-nonexpansive mapping, then $F(T)$ is a closed and convex subset of $C$. Besides, $T$ is said to be a firmly nonexpansive, if

$$
\begin{aligned}
& \|T x-T y\|^{2} \leq\langle x-y, T x-T y\rangle \quad \forall x, y \in C \\
& \quad \Leftrightarrow \quad\|T x-T y\|^{2} \leq\|x-y\|^{2}-\|(I-T) x-(I-T) y\|^{2} \quad \forall x, y \in C .
\end{aligned}
$$

Lemma 2.1 [22] Let $H$ be a real Hilbert space, and $\left\{x_{n}\right\}$ be a sequence in $H$. Then, for any given sequence $\left\{\lambda_{n}\right\}$ of positive numbers with $\sum_{i=1}^{\infty} \lambda_{n}=1$ for any positive integers $i, j$ with $i<j$ the following holds:

$$
\left\|\sum_{i=1}^{\infty} \lambda_{n} x_{n}\right\|^{2} \leq \sum_{i=1}^{\infty} \lambda_{n}\left\|x_{n}\right\|^{2}-\lambda_{i} \lambda_{j}\left\|x_{i}-x_{j}\right\|^{2} .
$$

Lemma 2.2 [23] Let $H$ be a real Hilbert space. For any $x, y \in H$, the following inequality holds:

$$
\|x+y\|^{2} \leq\|x\|^{2}+2\langle y, x+y\rangle
$$

Lemma 2.3 [24] Let $\left\{t_{n}\right\}$ be a sequence of real numbers. If there exists a subsequence $\left\{n_{i}\right\}$ of $\{n\}$ such that $t_{n_{i}}<t_{n_{i}+1}$ for all $i \geq 1$, then there exists a nondecreasing sequence $\{\tau(n)\}$ with $\tau(n) \rightarrow \infty$ such that for all (sufficiently large) positive integer number $n$, the following holds:

$$
t_{\tau(n)} \leq t_{\tau(n)+1}, \quad t_{n} \leq t_{\tau(n)+1} .
$$

In fact,

$$
\tau(n)=\max \left\{k \leq n: t_{k} \leq t_{k+1}\right\} .
$$

Definition 2.4 (Demiclosedness principle) Let $C$ be a nonempty closed convex subset of a real Hilbert space $H$, and $T: C \rightarrow C$ be a mapping with $F(T) \neq \emptyset$. Then $I-T$ is said to be demiclosed at zero, if for any sequence $\left\{x_{n}\right\} \subset C$ with $x_{n} \rightarrow x$ and $\left\|x_{n}-T x_{n}\right\| \rightarrow 0, x=T x$.

Remark 2.5 [25] It is well known that if $T: C \rightarrow C$ is a nonexpansive mapping, then $I-T$ is demiclosed at zero.

Lemma 2.6 Let $\left\{a_{n}\right\},\left\{b_{n}\right\}$ and $\left\{c_{n}\right\}$ be sequences of positive real numbers satisfying $a_{n+1} \leq$ $\left(1-b_{n}\right) a_{n}+c_{n}$ for all $n \geq 1$. If the following conditions are satisfied:

(1) $b_{n} \in(0,1)$ and $\sum_{n=1}^{\infty} b_{n}=\infty$,

(2) $\sum_{n=1}^{\infty} c_{n}<\infty$, or $\lim \sup _{n \rightarrow \infty} \frac{c_{n}}{b_{n}} \leq 0$, then $\lim _{n \rightarrow \infty} a_{n}=0$.

Lemma 2.7 [15] Let $H$ be a real Hilbert space, $B: H \rightarrow 2^{H}$ be a set-valued maximal monotone mapping, $\beta>0$, and let $J_{\beta}^{B}$ be the resolvent mapping of $B$ defined by $J_{\beta}^{B}:=(I+$ $\beta B)^{-1}$, then

(i) for each $\beta>0, J_{\beta}^{B}$ is a single-valued and firmly nonexpansive mapping; 
(ii) $D\left(J_{\beta}^{B}\right)=H$ and $F\left(J_{\beta}^{B}\right)=B^{-1}(0)$;

(iii) $\left(I-J_{\beta}^{B}\right)$ is a firmly nonexpansive mapping for each $\beta>0$;

(iv) suppose that $B^{-1}(0) \neq \emptyset$, then for each $x \in H$, each $x^{*} \in B^{-1}(0)$ and each $\beta>0$

$$
\left\|x-J_{\beta}^{B} x\right\|^{2}+\left\|J_{\beta}^{B} x-x^{*}\right\| \leq\left\|x-x^{*}\right\|^{2}
$$

(v) suppose that $B^{-1}(0) \neq \emptyset$. Then $\left\langle x-J_{\beta}^{B} x, J_{\beta}^{B} x-w\right\rangle \geq 0$ for each $x \in H$, each $w \in B^{-1}(0)$, and each $\beta>0$.

Lemma 2.8 Let $H_{1}, H_{2}$ be two real Hilbert spaces, $A: H_{1} \rightarrow H_{2}$ be a linear bounded operator and $A^{*}$ be the adjoint of $A$. Let $B: H_{2} \rightarrow 2^{H_{2}}$ be a set-valued maximal monotone mapping, $\beta>0$, and let $J_{\beta}^{B}$ be the resolvent mapping of $B$, then

(i) $\left\|\left(I-J_{\beta}^{B}\right) A x-\left(I-J_{\beta}^{B}\right) A y\right\|^{2} \leq\left\langle\left(I-J_{\beta}^{B}\right) A x-\left(I-J_{\beta}^{B}\right) A y, A x-A y\right\rangle$;

(ii) $\left\|A^{*}\left(I-J_{\beta}^{B}\right) A x-A^{*}\left(I-J_{\beta}^{B}\right) A y\right\|^{2} \leq\|A\|^{2}\left\langle\left(I-J_{\beta}^{B}\right) A x-\left(I-J_{\beta}^{B}\right) A y, A x-A y\right\rangle$;

(iii) if $\rho \in\left(0, \frac{2}{\|A\|^{2}}\right)$, then $\left(I-\rho A^{*}\left(I-J_{\beta}^{B}\right) A\right)$ is a nonexpansive mapping.

Proof By Lemma 2.7(iii), the mapping $\left(I-J_{\beta}^{B}\right)$ is firmly nonexpansive, hence the conclusions (i) and (ii) are obvious.

Now we prove the conclusion (iii).

In fact, for any $x, y \in H_{1}$, it follows from the conclusions (i) and (ii) that

$$
\begin{aligned}
\|(I- & \left.\rho A^{*}\left(I-J_{\beta}^{B}\right) A\right) x-\left(I-\rho A^{*}\left(I-J_{\beta}^{B}\right) A\right) y \|^{2} \\
= & \|x-y\|^{2}-2 \rho\left(x-y, A^{*}\left(I-J_{\beta}^{B}\right) A x-A^{*}\left(I-J_{\beta}^{B}\right) A y\right\rangle \\
& +\rho^{2}\left\|A^{*}\left(I-J_{\beta}^{B}\right) A x-A^{*}\left(I-J_{\beta}^{B}\right) A y\right\|^{2} \\
\leq & \|x-y\|^{2}-2 \rho\left(A x-A y,\left(I-J_{\beta}^{B}\right) A x-\left(I-J_{\beta}^{B}\right) A y\right\rangle \\
& +\rho^{2}\|A\|^{2}\left\|\left(I-J_{\beta}^{B}\right) A x-\left(I-J_{\beta}^{B}\right) A y\right\|^{2} \\
\leq & \|x-y\|^{2}-\rho\left(2-\rho\|A\|^{2}\right)\left\|\left(I-J_{\beta}^{B}\right) A x-\left(I-J_{\beta}^{B}\right) A y\right\|^{2} \\
\leq & \|x-y\|^{2} \quad\left(\text { since } \rho\left(2-\rho\|A\|^{2}\right) \geq 0\right) .
\end{aligned}
$$

This completes the proof of Lemma 2.8 .

\section{General split equality variational inclusion problem and strong convergence theorems}

Throughout this section we assume that

(1) $H_{1}, H_{2}, H_{3}$ are three real Hilbert spaces;

(2) $\left\{U_{i}\right\}_{i=1}^{\infty}: H_{1} \rightarrow 2^{H_{1}}$ and $\left\{K_{i}\right\}_{i=1}^{\infty}: H_{2} \rightarrow 2^{H_{2}}$ are two families of set-valued maximal monotone mappings, $\beta>0$ and $\gamma>0$ are given positive numbers;

(3) $A: H_{1} \rightarrow H_{3}$ and $B: H_{2} \rightarrow H_{3}$ are two bounded linear operators and $A^{*}, B^{*}$ are the adjoint of $A$ and $B$, respectively;

(4) $f=\left[\begin{array}{l}f_{1} \\ f_{2}\end{array}\right]$, where $f_{i}, i=1,2$ is a $k$-contractive mapping on $H_{i}$ with $k \in(0,1)$;

(5) the set of solutions of (GSEVIP) (1.5) $\Omega \neq \emptyset$,

$$
J_{\mu_{i}}^{\left(U_{i}, K_{i}\right)}:=\left[\begin{array}{c}
J_{\mu_{i}}^{U_{i}} \\
J_{\mu_{i}}^{K_{i}}
\end{array}\right], \quad G=[A-B], \quad G^{*}=\left[\begin{array}{c}
A^{*} \\
-B^{*}
\end{array}\right], \quad G^{*} G=\left[\begin{array}{cc}
A^{*} A & -A^{*} B \\
-B^{*} A & B^{*} B
\end{array}\right],
$$


(6) for any given $w_{0} \in H_{1} \times H_{2}$, the iterative sequence $\left\{w_{n}\right\} \subset H_{1} \times H_{2}$ is generated by

$$
w_{n+1}=\alpha_{n} w_{n}+\beta_{n} f\left(w_{n}\right)+\sum_{i=1}^{\infty} \gamma_{n, i}\left(J_{\mu_{i}}^{\left(U_{i}, K_{i}\right)}\left(I-\lambda_{n, i} G^{*} G\right) w_{n}\right), \quad n \geq 0
$$

or its equivalent form:

$$
\left\{\begin{array}{l}
x_{n+1}=\alpha_{n} x_{n}+\beta_{n} f_{1}\left(x_{n}\right)+\sum_{i=1}^{\infty} \gamma_{n, i}\left(J_{\mu_{i}}^{U_{i}}\left(x_{n}-\lambda_{n, i}\left(A^{*}\left(A x_{n}-B y_{n}\right)\right)\right)\right), \\
y_{n+1}=\alpha_{n} y_{n}+\beta_{n} f_{2}\left(y_{n}\right)+\sum_{i=1}^{\infty} \gamma_{n, i}\left(J_{\mu_{i}}^{K i}\left(y_{n}+\lambda_{n, i}\left(B^{*}\left(A x_{n}-B y_{n}\right)\right)\right)\right),
\end{array}\right.
$$

where $\left\{\alpha_{n}\right\},\left\{\beta_{n}\right\},\left\{\gamma_{n, i}\right\}$ are the sequences of nonnegative numbers satisfying

$$
\alpha_{n}+\beta_{n}+\sum_{i=1}^{\infty} \gamma_{n, i}=1, \quad \text { for each } n \geq 0
$$

We are now in a position to give the following results.

Lemma 3.1 Let $H_{1}, H_{2}, H_{3}, A, B, A^{*}, B^{*},\left\{U_{i}\right\},\left\{K_{i}\right\}, J_{\mu_{i}}^{\left(U_{i}, K_{i}\right)}, G, G^{*}$ be the same as above. If $\Omega \neq \emptyset$ (the solution set of (GSEVIP) (1.5)), then $w^{*}:=\left(x^{*}, y^{*}\right) \in H_{1} \times H_{2}$ is a solution of (GSEVIP) (1.5) if and only iffor each $i \geq 1$, and for any given $\gamma>0$ and $\mu>0$

$$
w^{*}=J_{\mu}^{\left(U_{i}, K_{i}\right)}\left(I-\gamma G^{*} G\right) w^{*} .
$$

Proof Indeed, if $w^{*}=\left(x^{*}, y^{*}\right) \in H_{1} \times H_{2}$ is a solution of (GSEVIP) (1.5), then by Lemma 2.7(ii), for each $i \geq 1$, and for any $\gamma>0$ and $\mu>0$ we have

$$
\begin{gathered}
x^{*} \in U_{i}^{-1}(0)=F\left(J_{\mu}^{U_{i}}\right), \quad y^{*} \in K_{i}^{-1}(0)=F\left(J_{\mu}^{K_{i}}\right) \quad \text { and } A x^{*}=B y^{*} \\
\Leftrightarrow \quad x^{*}=J_{\mu}^{U_{i}} x^{*}, \quad y^{*}=J_{\mu}^{K_{i}} y^{*} \quad \text { and } A x^{*}=B y^{*} .
\end{gathered}
$$

Hence we have $G\left(w^{*}\right)=A x^{*}-B y^{*}=0$, and so

$$
J_{\mu_{i}}^{\left(U_{i}, K_{i}\right)}\left(I-\gamma G^{*} G\right)\left(w^{*}\right)=J_{\mu}^{\left(U_{i}, K_{i}\right)}\left(w^{*}\right)=\left(J_{\mu}^{U_{i}} x^{*}, J_{\mu}^{K_{i}} y^{*}\right)=w^{*}
$$

This implies that (3.2) is true.

Conversely, if $w^{*}=\left(x^{*}, y^{*}\right) \in H_{1} \times H_{2}$ satisfies (3.2), then we have

$$
\left\{\begin{array}{l}
x^{*}=J_{\mu}^{U_{i}}\left[x^{*}-\gamma A^{*}\left(A x^{*}-B y^{*}\right)\right] \\
y^{*}=J_{\mu}^{K_{i}}\left[y^{*}+\gamma B^{*}\left(A x^{*}-B y^{*}\right)\right]
\end{array}\right.
$$

We make the assumption that the solution set $\Omega$ of (GSEVIP) (1.5) is nonempty. Hence the sets $U_{i}^{-1}(0)$ and $K_{i}^{-1}(0)$ both are nonempty. By Lemma 2.7(v) and (3.3), we have

$$
\left\langle x^{*}-\left(x^{*}-\gamma A^{*}\left(A x^{*}-B y^{*}\right)\right), x-x^{*}\right\rangle \geq 0, \quad \forall x \in U_{i}^{-1}(0),
$$

and so

$$
\left\langle A x^{*}-B y^{*}, A x-A x^{*}\right\rangle \geq 0, \quad \forall x \in U_{i}^{-1}(0) .
$$


Similarly, by Lemma $2.7(\mathrm{v})$ and (3.3) again, one gets

$$
\left\langle A x^{*}-B y^{*}, B y^{*}-B y\right\rangle \geq 0, \quad \forall y \in K_{i}^{-1}(0) .
$$

Adding up (3.4) and (3.5), we have

$$
\left\langle A x^{*}-B y^{*}, A x-A x^{*}+B y^{*}-B y\right| \geq 0, \quad \forall x \in U_{i}^{-1}(0) \text { and } y \in K_{i}^{-1}(0) .
$$

Simplifying it, we have

$$
\left\|A x^{*}-B y^{*}\right\|^{2} \leq\left\langle A x^{*}-B y^{*}, A x-B y\right\rangle, \quad \forall x \in U_{i}^{-1}(0) \text { and } y \in K_{i}^{-1}(0) .
$$

Since $\Omega \neq \emptyset$, taking $\bar{w}=(\bar{x}, \bar{y}) \in \Omega$, for each $i \geq 1$, we have $\bar{x} \in U_{i}^{-1}(0)$ and $\bar{y} \in K_{i}^{-1}(0)$ and $A \bar{x}=B \bar{y}$. In (3.6), taking $x=\bar{x}$ and $y=\bar{y}$, we have

$$
\left\|A x^{*}-B y^{*}\right\|=0, \text { i.e., } A x^{*}=B y^{*} .
$$

Hence from (3.3) and (3.7)

$$
\left\{\begin{array}{l}
x^{*}=J_{\mu}^{U_{i}}\left(x^{*}\right), \\
y^{*}=J_{\mu}^{K_{i}}\left(y^{*}\right),
\end{array} \quad \Leftrightarrow \quad 0 \in U_{i}\left(x^{*}\right), \quad 0 \in K_{i}\left(y^{*}\right), \quad \forall i \geq 1 .\right.
$$

It follows from (3.7) and (3.8) that $w^{*}$ is a solution of (GSEVIP) (1.5).

This completes the proof of Lemma 3.1.

Lemma 3.2 If $\lambda \in\left(0, \frac{2}{L}\right)$, where $L=\|G\|^{2}$, then $\left(I-\lambda G^{*} G\right): H_{1} \times H_{2} \rightarrow H_{1} \times H_{2}$ is a nonexpansive mapping.

Proof In fact, for any $w, u \in H_{1} \times H_{2}$, we have

$$
\begin{aligned}
\| & \left(I-\lambda G^{*} G\right) u-\left(I-\lambda G^{*} G\right) w \|^{2} \\
& =\left\|(u-w)-\lambda G^{*} G(u-w)\right\|^{2} \\
& =\|u-w\|^{2}+\lambda^{2}\left\|G^{*} G(u-w)\right\|^{2}-2 \lambda\left\langle u-w, G^{*} G(u-w)\right\rangle \\
& \leq\|u-w\|^{2}+\lambda^{2} L\|G(u-w)\|^{2}-2 \lambda\langle G(u-w), G(u-w)\rangle \\
& =\|u-w\|^{2}+\lambda^{2} L\|G(u-w)\|^{2}-2 \lambda\|G(u-w)\|^{2} \\
& =\|u-w\|^{2}-\lambda(2-\lambda L)\|G(u-w)\|^{2} \\
& \leq\|u-w\|^{2} .
\end{aligned}
$$

This completes the proof.

Theorem 3.3 Let $H_{1}, H_{2}, H_{3}, A, B, A^{*}, B^{*},\left\{U_{i}\right\},\left\{K_{i}\right\}, J_{\mu_{i}}^{\left(U_{i}, K_{i}\right)}, G, G^{*}, f$ be the same as above. Let $\left\{w_{n}\right\}$ be the sequence defined by (3.1). If the solution set $\Omega$ of (GSEVIP) (1.5) is nonempty and the following conditions are satisfied:

(i) $\alpha_{n}+\beta_{n}+\sum_{i=1}^{\infty} \gamma_{n, i}=1$, for each $n \geq 0$;

(ii) $\lim _{n \rightarrow \infty} \beta_{n}=0$, and $\sum_{n=0}^{\infty} \beta_{n}=\infty$; 
(iii) $\liminf _{n \rightarrow \infty} \alpha_{n} \gamma_{n, i}>0$ for each $i \geq 1$;

(iv) $\left\{\lambda_{n, i}\right\} \subset\left(0, \frac{2}{L}\right)$ for each $i \geq 1$, where $L=\|G\|^{2}$,

then the sequence $\left\{w_{n}\right\}$ converges strongly to $w^{*}=P_{\Omega_{2}} f\left(w^{*}\right)$, which is a solution of (GSEVIP) (1.5).

Proof (I) First we prove that the sequence $\left\{w_{n}\right\}$ is bounded.

In fact, for any given $z \in \Omega$, it follows from Lemma 3.1, Lemma 3.2, and condition (iv) that

$$
z=J_{\mu}^{\left(U_{i}, K_{i}\right)}\left(I-\lambda_{n, i} G^{*} G\right) z, \quad \text { for each } i \geq 1,
$$

and $\left(I-\lambda_{n, i} G^{*} G\right): H_{1} \times H_{2} \rightarrow H_{1} \times H_{2}$ is a nonexpansive mapping. Also by Lemma 2.7(i), for each $i \geq 1, J_{\mu_{i}}^{\left(U_{i}, K_{i}\right)}$ is a firmly nonexpansive mapping. Hence we have

$$
\begin{aligned}
\left\|w_{n+1}-z\right\|= & \left\|\left(\alpha_{n} w_{n}+\beta_{n} f\left(w_{n}\right)+\sum_{i=1}^{\infty} \gamma_{n, j} J_{\mu_{i}}^{\left(U_{i}, K_{i}\right)}\left(I-\lambda_{n, i} G^{*} G\right) w_{n}\right)-z\right\| \\
\leq & \alpha_{n}\left\|w_{n}-z\right\|+\beta_{n}\left\|f\left(w_{n}\right)-z\right\|+\sum_{i=1}^{\infty} \gamma_{n, i}\left\|f_{\mu_{i}}^{\left(U_{i}, K_{i}\right)}\left(I-\lambda_{n, i} G^{*} G\right) w_{n}-z\right\| \\
\leq & \alpha_{n}\left\|w_{n}-z\right\|+\beta_{n}\left\|f\left(w_{n}\right)-z\right\|+\sum_{i=1}^{\infty} \gamma_{n, i}\left\|\left(I-\lambda_{n, i} G^{*} G\right) w_{n}-z\right\| \\
\leq & \alpha_{n}\left\|w_{n}-z\right\|+\beta_{n}\left\|f\left(w_{n}\right)-z\right\| \\
& +\sum_{i=1}^{\infty} \gamma_{n, i}\left\|\left(I-\lambda_{n, i} G^{*} G\right) w_{n}-\left(I-\lambda_{n, i} G^{*} G\right) z\right\| \\
\leq & \alpha_{n}\left\|w_{n}-z\right\|+\beta_{n}\left\|f\left(w_{n}\right)-z\right\|+\sum_{i=1}^{\infty} \gamma_{n, i}\left\|w_{n}-z\right\| \\
= & \left(1-\beta_{n}\right)\left\|w_{n}-z\right\|+\beta_{n}\left\|f\left(w_{n}\right)-z\right\| \\
\leq & \left(1-\beta_{n}\right)\left\|w_{n}-z\right\|+\beta_{n}\left\|f\left(w_{n}\right)-f(z)\right\|+\beta_{n}\|f(z)-z\| \\
\leq & \left(1-\beta_{n}\right)\left\|w_{n}-z\right\|+k \beta_{n}\left\|w_{n}-z\right\|+\beta_{n}\|f(z)-z\| \\
= & \left(1-(1-k) \beta_{n}\right)\left\|w_{n}-z\right\|+(1-k) \beta_{n} \frac{1}{1-k}\|f(z)-z\| \\
\leq & \max \left\{\left\|w_{n}-z\right\|, \frac{1}{1-k}\|f(z)-z\|\right\} .
\end{aligned}
$$

By induction, we can prove that

$$
\left\|w_{n}-z\right\| \leq \max \left\{\left\|w_{0}-z\right\|, \frac{1}{1-k}\|f(z)-z\|\right\}, \quad \forall n \geq 0 .
$$

This shows that $\left\{w_{n}\right\}$ is bounded, and so is $\left\{f\left(w_{n}\right)\right\}$.

(II) Now we prove that the following inequality holds:

$$
\begin{aligned}
& \alpha_{n} \gamma_{n, i}\left\|w_{n}-J_{\mu_{i}}^{\left(U_{i} K_{i}\right)}\left(I-\lambda_{n, i} G^{*} G\right) w_{n}\right\|^{2} \\
& \quad \leq\left\|w_{n}-z\right\|^{2}-\left\|w_{n+1}-z\right\|^{2}+\beta_{n}\left\|f\left(w_{n}\right)-z\right\|^{2} \quad \text { for each } i \geq 1 .
\end{aligned}
$$


Indeed, it follows from (3.1) and Lemma 2.1 that for each $i \geq 1$

$$
\begin{aligned}
\left\|w_{n+1}-z\right\|^{2}= & \left\|\alpha_{n}\left(w_{n}-z\right)+\beta_{n}\left(f\left(w_{n}\right)-z\right)+\sum_{j=1}^{\infty} \gamma_{n, j}\left(J_{\mu_{j}}^{\left(U_{j}, K_{j}\right)}\left(I-\lambda_{n, j} G^{*} G\right) w_{n}-z\right)\right\|^{2} \\
\leq & \alpha_{n}\left\|w_{n}-z\right\|^{2}+\beta_{n}\left\|f\left(w_{n}\right)-z\right\|^{2}+\sum_{j=1}^{\infty} \gamma_{n, j}\left\|J_{\mu_{j}}^{\left(U_{j}, K_{j}\right)}\left(I-\lambda_{n, j} G^{*} G\right) w_{n}-z\right\|^{2} \\
& -\alpha_{n} \gamma_{n, i}\left\|w_{n}-J_{\mu_{i}}^{\left(U_{i}, K_{i}\right)}\left(I-\lambda_{n, i} G^{*} G\right) w_{n}\right\|^{2} \\
\leq & \alpha_{n}\left\|w_{n}-z\right\|^{2}+\beta_{n}\left\|f\left(w_{n}\right)-z\right\|^{2}+\sum_{j=1}^{\infty} \gamma_{n, j}\left\|w_{n}-z\right\|^{2} \\
& -\alpha_{n} \gamma_{n, i}\left\|w_{n}-J_{\mu_{i}}^{\left(U_{i}, K_{i}\right)}\left(I-\lambda_{n, i} G^{*} G\right) w_{n}\right\|^{2} \\
= & \left(1-\beta_{n}\right)\left\|w_{n}-z\right\|^{2}+\beta_{n}\left\|f\left(w_{n}\right)-z\right\|^{2} \\
& -\alpha_{n} \gamma_{n, i}\left\|w_{n}-J_{\mu_{i}}^{\left(U_{i}, K_{i}\right)}\left(I-\lambda_{n, i} G^{*} G\right) w_{n}\right\|^{2} .
\end{aligned}
$$

This implies that for each $i \geq 1$

$$
\alpha_{n} \gamma_{n, i}\left\|w_{n}-J_{\mu_{i}}^{\left(U_{i}, K_{i}\right)}\left(I-\lambda_{n, i} G^{*} G\right) w_{n}\right\|^{2} \leq\left\|w_{n}-z\right\|^{2}-\left\|w_{n+1}-z\right\|^{2}+\beta_{n}\left\|f\left(w_{n}\right)-z\right\|^{2} .
$$

Inequality (3.3) is proved.

It is easy to see that the solution set $\Omega$ of (GSEVIP) (1.5) is a closed and convex subset in $H_{1} \times H_{2}$. By the assumption that $\Omega$ is nonempty, so it is a nonempty closed and convex subset in $H_{1} \times H_{2}$. Hence the metric projection $P_{\Omega}$ is well defined. In addition, since $P_{\Omega} f$ : $H_{1} \times H_{2} \rightarrow \Omega$ is a contractive mapping, there exists a unique $w^{*} \in \Omega$ such that

$$
w^{*}=P_{\Omega} f\left(w^{*}\right) .
$$

(III) Now we prove that $\left\{w_{n}\right\}$ converges strongly to $w^{*}$.

For the purpose, we consider two cases.

Case I. Suppose that the sequence $\left\{\left\|w_{n}-w^{*}\right\|\right\}$ is monotone. Since $\left\{\left\|w_{n}-w^{*}\right\|\right\}$ is bounded, $\left\{\left\|w_{n}-w^{*}\right\|\right\}$ is convergent. Since $w^{*} \in \Omega$, in (3.9) taking $z=w^{*}$ and letting $n \rightarrow \infty$, in view of conditions (ii) and (iii), we have

$$
\lim _{n \rightarrow \infty}\left\|w_{n}-J_{\mu_{i}}^{\left(U_{i}, K_{i}\right)}\left(I-\lambda_{n, i} G^{*} G\right) w_{n}\right\|=0, \quad \text { for each } i \geq 1
$$

On the other hand, by Lemma 2.2 and (3.1), we have

$$
\begin{aligned}
\left\|w_{n+1}-w^{*}\right\|^{2}= & \left\|\left(\alpha_{n} w_{n}+\beta_{n} f\left(w_{n}\right)+\sum_{i=1}^{\infty} \gamma_{n, i} J_{\mu_{i}}^{\left(U_{i}, K_{i}\right)}\left(I-\lambda_{n, i} G^{*} G\right) w_{n}\right)-w^{*}\right\|^{2} \\
= & \| \alpha_{n}\left(w_{n}-w^{*}\right)+\beta_{n}\left(f\left(w_{n}\right)-w^{*}\right) \\
& +\sum_{i=1}^{\infty} \gamma_{n, i}\left(J_{\mu_{i}}^{\left(U_{i} K_{i}\right)}\left(I-\lambda_{n, i} G^{*} G\right) w_{n}-w^{*}\right) \|^{2}
\end{aligned}
$$




$$
\begin{aligned}
\leq & \left\|\alpha_{n}\left(w_{n}-w^{*}\right)+\sum_{i=1}^{\infty} \gamma_{n, i}\left(J_{\mu_{i}}^{\left(U_{i}, K_{i}\right)}\left(I-\lambda_{n, i} G^{*} G\right) w_{n}-w^{*}\right)\right\|^{2} \\
& +2 \beta_{n}\left\langle f\left(w_{n}\right)-w^{*}, w_{n+1}-w^{*}\right\rangle \quad(\text { by Lemma 2.2) } \\
\leq & \left\{\alpha_{n}\left\|w_{n}-w^{*}\right\|+\sum_{i=1}^{\infty} \gamma_{n, i}\left\|w_{n}-w^{*}\right\|\right\}^{2} \\
& +2 \beta_{n}\left\langle f\left(w_{n}\right)-f\left(w^{*}\right), w_{n+1}-w^{*}\right\rangle+2 \beta_{n}\left\langle f\left(w^{*}\right)-w^{*}, w_{n+1}-w^{*}\right\rangle \\
= & \left(1-\beta_{n}\right)^{2}\left\|w_{n}-w^{*}\right\|^{2}+2 \beta_{n} k\left\|w_{n}-w^{*}\right\|\left\|w_{n+1}-w^{*}\right\| \\
& +2 \beta_{n}\left\langle f\left(w^{*}\right)-w^{*}, w_{n+1}-w^{*}\right\rangle \\
\leq & \left(1-\beta_{n}\right)^{2}\left\|w_{n}-w^{*}\right\|^{2}+\beta_{n} k\left\{\left\|w_{n}-w^{*}\right\|^{2}+\left\|w_{n+1}-w^{*}\right\|^{2}\right\} \\
& +2 \beta_{n}\left\langle f\left(w^{*}\right)-w^{*}, w_{n+1}-w^{*}\right\rangle .
\end{aligned}
$$

Simplifying it we have

$$
\begin{aligned}
\left\|w_{n+1}-w^{*}\right\|^{2} \leq & \frac{\left(1-\beta_{n}\right)^{2}+\beta_{n} k}{1-\beta_{n} k}\left\|w_{n}-w^{*}\right\|^{2}+\frac{2 \beta_{n}}{1-\beta_{n} k}\left\langle f\left(w^{*}\right)-w^{*}, w_{n+1}-w^{*}\right\rangle \\
= & \frac{1-2 \beta_{n}+\beta_{n} k}{1-\beta_{n} k}\left\|w_{n}-w^{*}\right\|^{2}+\frac{\beta_{n}^{2}}{1-\beta_{n} k}\left\|w_{n}-w^{*}\right\|^{2} \\
& +\frac{2 \beta_{n}}{1-\beta_{n} k}\left\langle f\left(w^{*}\right)-w^{*}, w_{n+1}-w^{*}\right\rangle \\
= & \left(1-\frac{2(1-k) \beta_{n}}{1-\beta_{n} k}\right)\left\|w_{n}-w^{*}\right\|^{2} \\
& +\frac{2(1-k) \beta_{n}}{1-\beta_{n} k}\left\{\frac{\beta_{n} M}{2(1-k)}+\frac{1}{1-k}\left\langle f\left(w^{*}\right)-w^{*}, w_{n+1}-w^{*}\right\rangle\right\} \\
= & \left(1-\eta_{n}\right)\left\|w_{n}-w^{*}\right\|^{2}+\eta_{n} \delta_{n},
\end{aligned}
$$

where

$$
\begin{aligned}
& \eta_{n}=\frac{2(1-k) \beta_{n}}{1-\beta_{n} k}, \quad \delta_{n}=\frac{\beta_{n} M}{2(1-k)}+\frac{1}{1-k}\left\langle f\left(w^{*}\right)-w^{*}, w_{n+1}-w^{*}\right\rangle, \\
& M=\sup _{n \geq 0}\left\|w_{n}-w^{*}\right\|^{2} .
\end{aligned}
$$

By condition (ii), $\lim _{n \rightarrow \infty} \beta_{n}=0$ and $\sum_{n=1}^{\infty} \beta_{n}=\infty$, and so is $\sum_{n=1}^{\infty} \eta_{n}=\infty$.

Next we prove that

$$
\limsup _{n \rightarrow \infty} \delta_{n} \leq 0
$$

In fact, since $\left\{w_{n}\right\}$ is bounded in $H_{1} \times H_{2}$, there exists a subsequence $\left\{w_{n_{k}}\right\} \subset\left\{w_{n}\right\}$ with $w_{n_{k}} \rightarrow v^{*}$ (some point in $\left.C \times Q\right)$, and $\lambda_{n_{k}, i} \rightarrow \lambda_{i} \in\left(0, \frac{2}{L}\right)$ such that

$$
\lim _{n \rightarrow \infty}\left\langle f\left(w^{*}\right)-w^{*}, w_{n_{k}}-w^{*}\right\rangle=\limsup _{n \rightarrow \infty}\left\langle f\left(w^{*}\right)-w^{*}, w_{n}-w^{*}\right\rangle
$$


Since

$$
\left\|w_{n_{k}}-J_{\mu_{i}}^{\left(U_{i}, K_{i}\right)}\left(I-\lambda_{n_{k}, i} G^{*} G\right) w_{n_{k}}\right\| \rightarrow 0, \quad \text { for each } i \geq 1
$$

and $J_{\mu_{i}}^{\left(U_{i}, K_{i}\right)}\left(I-\lambda_{n_{k}, i} G^{*} G\right)$ is a nonexpansive mapping, by Remark $2.5, I-J_{\mu_{i}}^{\left(B_{i}, K_{i}\right)}\left(I-\lambda_{n, i} G^{*} G\right)$ is demiclosed at zero, hence we have

$$
v^{*}=J_{\mu_{i}}^{\left(U_{i}, K_{i}\right)}\left(I-\lambda_{n, i} G^{*} G\right) v^{*}, \quad \forall i \geq 1
$$

By Lemma 3.1, this implies that $v^{*} \in \Omega$. In addition, since $w^{*}=P_{\Omega} f\left(w^{*}\right)$, we have

$$
\begin{aligned}
\limsup _{n \rightarrow \infty}\left\langle f\left(w^{*}\right)-w^{*}, w_{n}-w^{*}\right\rangle & =\lim _{n \rightarrow \infty}\left\langle f\left(w^{*}\right)-w^{*}, w_{n_{k}}-w^{*}\right\rangle \\
& =\left\langle f\left(w^{*}\right)-w^{*}, v^{*}-w^{*}\right\rangle \leq 0 .
\end{aligned}
$$

This shows that (3.13) is true. Taking $a_{n}=\left\|w_{n}-w^{*}\right\|^{2}, b_{n}=\eta_{n}$, and $c_{n}=\delta_{n} \eta_{n}$ in Lemma 2.6, therefore all conditions in Lemma 2.6 are satisfied. We have $w_{n} \rightarrow w^{*}$.

Case II. If the sequence $\left\{\left\|w_{n}-w^{*}\right\|\right\}$ is not monotone, by Lemma 2.3, there exists a sequence of positive integers: $\{\tau(n)\}, n \geq n_{0}$ (where $n_{0}$ large enough) such that

$$
\tau(n)=\max \left\{k \leq n:\left\|w_{k}-w^{*}\right\| \leq\left\|w_{k+1}-w^{*}\right\|\right\} .
$$

Clearly $\{\tau(n)\}$ is a nondecreasing, $\tau(n) \rightarrow \infty$ as $n \rightarrow \infty$, and for all $n \geq n_{0}$

$$
\left\|w_{\tau(n)}-w^{*}\right\| \leq\left\|w_{\tau(n)+1}-w^{*}\right\| ; \quad\left\|w_{n}-w^{*}\right\| \leq\left\|w_{\tau(n)+1}-w^{*}\right\| .
$$

Therefore $\left\{\left\|w_{\tau(n)}-w^{*}\right\|\right\}$ is a nondecreasing sequence. According to Case I, $\lim _{n \rightarrow \infty} \| w_{\tau(n)}-$ $w^{*} \|=0$ and $\lim _{n \rightarrow \infty}\left\|w_{\tau(n)+1}-w^{*}\right\|=0$. Hence we have

$$
0 \leq\left\|w_{n}-w^{*}\right\| \leq \max \left\{\left\|w_{n}-w^{*}\right\|,\left\|w_{\tau(n)}-w^{*}\right\|\right\} \leq\left\|w_{\tau(n)+1}-w^{*}\right\| \rightarrow 0, \quad \text { as } n \rightarrow \infty
$$

This implies that $w_{n} \rightarrow w^{*}$ and $w^{*}=P_{\Omega} f\left(w^{*}\right)$ is a solution of (GSEVIP) (1.5).

This completes the proof of Theorem 3.3.

Remark 3.4 Theorem 3.3 extends and improves the main results in Moudafi et al. [11, 12, 19], Eslamian and Latif [13], Chen et al. [14], Chuang [15], Naraghirad [21] and Ansari and Rehan [17].

\section{General split equality optimization problem and strong convergence theorems}

Let $H_{1}, H_{2}$, and $H_{3}$ be three real Hilbert spaces. Let $A: H_{1} \rightarrow H_{3}$ and $B: H_{2} \rightarrow H_{3}$ be two linear and bounded operators. The so-called general split equality optimization problem (GSEOP) is to find $x^{*} \in H_{1}$, and $y^{*} \in H_{2}$ such that for each $i \geq 1$

$$
h_{i}\left(x^{*}\right)=\min _{x \in H_{1}} h_{i}(x), \quad g_{i}\left(y^{*}\right)=\min _{z \in H_{2}} g_{i}(z) \quad \text { and } \quad A x^{*}=B y^{*},
$$


where $h_{i}: H_{1} \rightarrow \mathbb{R}$ and $g_{i}: H_{2} \rightarrow \mathbb{R}$ are two families of proper, lower semicontinuous, and convex functions.

For each $i \geq 1$ denote by $\partial h_{i}=U_{i}$ and $\partial g_{i}=K_{i}$. Then the mappings $U_{i}: H_{1} \rightarrow 2^{H_{1}}$ and $K_{i}: H_{2} \rightarrow 2^{H_{2}}, i=1,2, \ldots$ both are set-valued maximal monotone mappings, and

$$
\begin{aligned}
& h_{i}\left(x^{*}\right)=\min _{x \in H_{1}} h_{i}(x) \quad \Leftrightarrow \quad 0 \in \partial h_{i}\left(x^{*}\right)=U_{i}\left(x^{*}\right), \\
& g_{i}\left(y^{*}\right)=\min _{z \in H_{2}} g_{i}(z) \quad \Leftrightarrow \quad 0 \in \partial g_{i}\left(y^{*}\right)=K_{i}\left(y^{*}\right) .
\end{aligned}
$$

Therefore (GSEOP) (4.1) is equivalent to the following general split equality variational inclusion problem (GSEVIP): to find $x^{*} \in H_{1}$ and $y^{*} \in H_{2}$ such that

$$
0 \in \bigcap_{i=1}^{\infty} U_{i}\left(x^{*}\right), \quad 0 \in \bigcap_{i=1}^{\infty} K_{i}\left(y^{*}\right) \quad \text { and } \quad A x^{*}=B y^{*} .
$$

Therefore, the following theorem can be obtained from Theorem 3.3 immediately.

Theorem 4.1 Let $H_{1}, H_{2}, H_{3}, A, B, A^{*}, B^{*},\left\{U_{i}\right\},\left\{K_{i}\right\}$ be the same as above. Let $J_{\mu_{i}}^{\left(U_{i}, K_{i}\right)}, G$, $G^{*}, f$ be the same as in Theorem 3.3. Let $\left\{w_{n}\right\}$ be the sequence defined by (3.1). If the solution set $\Omega_{1}$ of (GSEVIP) (4.1) is nonempty and the following conditions are satisfied:

(i) $\alpha_{n}+\beta_{n}+\sum_{i=1}^{\infty} \gamma_{n, i}=1$, for each $n \geq 0$;

(ii) $\lim _{n \rightarrow \infty} \beta_{n}=0$, and $\sum_{n=0}^{\infty} \beta_{n}=\infty$;

(iii) $\liminf _{n \rightarrow \infty} \alpha_{n} \gamma_{n, i}>0$ for each $i \geq 1$;

(iv) $\left\{\lambda_{n, i}\right\} \subset\left(0, \frac{2}{L}\right)$ for each $i \geq 1$, where $L=\|G\|^{2}$,

then the sequence $\left\{w_{n}\right\}$ converges strongly to $w^{*}=P_{\Omega_{1}} f\left(w^{*}\right)$, which is a solution of (GSEOP) (4.1).

By using Theorem 3.3 and Theorem 4.1, now we give some corollaries for the split equality feasibility problem, the split equality equilibrium problem, and the split optimization problem.

Let $H_{1}, H_{2}, H_{3}, C, Q, A, B$ be the same as in the split equality feasibility problem (1.3). Let $i_{C}$ and $i_{Q}$ be the indicator function of $C$ and $Q$, respectively, defined by (1.7). In Theorem 4.1, take $\{U\}=\left\{\partial i_{C}\right\},\{K\}=\left\{\partial i_{Q}\right\}$, and $J_{\mu}^{(U, K)}=P_{C \times Q}:=\left[\begin{array}{c}P_{C} \\ P_{Q}\end{array}\right]$, therefore we have the following.

Corollary 4.2 Let $H_{1}, H_{2}, H_{3}, A, B, A^{*}, B^{*}, P_{C \times Q}$ be the same as above. Let $G, G^{*}, f$ be the same as in Theorem 4.1. Let $\left\{w_{n}\right\}$ be the sequence generated by $w_{0} \in H_{1} \times H_{2}$

$$
w_{n+1}=\alpha_{n} w_{n}+\beta_{n} f\left(w_{n}\right)+\gamma_{n}\left(P_{C \times Q}\left(I-\lambda_{n} G^{*} G\right) w_{n}\right), \quad n \geq 0,
$$

or its equivalent form

$$
\left\{\begin{array}{l}
x_{n+1}=\alpha_{n} x_{n}+\beta_{n} f_{1}\left(x_{n}\right)+\gamma_{n}\left(P_{C}\left(x_{n}-\lambda_{n}\left(A^{*}\left(A x_{n}-B y_{n}\right)\right)\right)\right), \\
y_{n+1}=\alpha_{n} y_{n}+\beta_{n} f_{2}\left(y_{n}\right)+\gamma_{n}\left(P_{Q}\left(y_{n}+\lambda_{n}\left(B^{*}\left(A x_{n}-B y_{n}\right)\right)\right)\right) .
\end{array}\right.
$$

If the solution set $\Gamma_{1}$ of (SEFP) (1.3) is nonempty and the following conditions are satisfied:

(i) $\alpha_{n}+\beta_{n}+\gamma_{n}=1$, for each $n \geq 0$; 
(ii) $\lim _{n \rightarrow \infty} \beta_{n}=0$, and $\sum_{n=0}^{\infty} \beta_{n}=\infty$;

(iii) $\liminf _{n \rightarrow \infty} \alpha_{n} \gamma_{n}>0$;

(iv) $\left\{\lambda_{n}\right\} \subset\left(0, \frac{2}{L}\right)$ for each $i \geq 1$, where $L=\|G\|^{2}$,

then the sequence $\left\{w_{n}\right\}$ converges strongly to $w^{*}=P_{\Gamma_{1}} f\left(w^{*}\right)$, which is a solution of (SEFP) (1.3).

Remark 4.3 Since the simultaneous iterative sequence $\left\{\left(x_{n}, y_{n}\right)\right\}(4.4)$ converges strongly to a solution of (SEFP) (1.3). Therefore it provides an affirmative answer to Moudafi's open question 1.1 [11].

Let $h, g: D \times D \rightarrow(-\infty,+\infty)$ be two equilibrium functions. For given $\lambda>0$, let $R_{\lambda, h}$ and $R_{\lambda, g}$ be the resolvents of $h$ and $g$ (defined by (1.9)), respectively.

The so-called split equality equilibrium problem with respective to $h, g$, and $D(\operatorname{SEEP}(h$, $g, D))$ is to find $x^{*} \in D, y^{*} \in D$ such that

$$
h\left(x^{*}, u\right) \geq 0, \quad \forall u \in D, \quad g\left(y^{*}, v\right) \geq 0, \quad \forall v \in D \quad \text { and } \quad A x^{*}=B y^{*}
$$

where $A, B: D \rightarrow D$ are two linear and bounded operators.

By Proposition 1.2, the $(\operatorname{SEEP}(h, g, D))(4.5)$ is equivalent to find $x^{*} \in D, y^{*} \in D$ such that for each $\lambda>0$

$$
\begin{aligned}
x^{*} & \in E P(h, D), \quad y^{*} \in E P(g, D) \quad \text { and } A x^{*}=B y^{*} \\
& \Leftrightarrow \quad x^{*} \in F\left(R_{\lambda h}\right), \quad y^{*} \in F\left(R_{\lambda g}\right) \text { and } A x^{*}=B y^{*} .
\end{aligned}
$$

Letting $C=F\left(R_{\lambda h}\right), Q=F\left(R_{\lambda g}\right)$, by Proposition 1.2, $C$ and $Q$ both are nonempty closed and convex subset of $D$. Hence the problem (4.5) (and so the problem (4.6)) is equivalent to the following split equality feasibility problem:

$$
\text { to find } x^{*} \in C, y^{*} \in Q \text { such that } A x^{*}=B y^{*} \text {. }
$$

In Corollary 4.2 taking $H_{1}=H_{2}=H_{3}=D$, from Corollary 4.2 we have the following.

Corollary 4.4 Let $D, C, Q$ be the same as above. Let $A, B, A^{*}, B^{*}, P_{C \times Q}, G, G^{*}, f$ be the same as in Corollary 4.2. For any given $w_{0} \in D \times D$, let $\left\{w_{n}\right\}$ be the sequence generated by

$$
w_{n+1}=\alpha_{n} w_{n}+\beta_{n} f\left(w_{n}\right)+\gamma_{n}\left(P_{C \times Q}\left(I-\lambda_{n} G^{*} G\right) w_{n}\right), \quad n \geq 0 .
$$

If the solution set $\Gamma_{2}$ of $(\operatorname{SEEP}(h, g, D))(4.5)$ is nonempty and the following conditions are satisfied:

(i) $\alpha_{n}+\beta_{n}+\gamma_{n}=1$, for each $n \geq 0$;

(ii) $\lim _{n \rightarrow \infty} \beta_{n}=0$, and $\sum_{n=0}^{\infty} \beta_{n}=\infty$;

(iii) $\liminf _{n \rightarrow \infty} \alpha_{n} \gamma_{n}>0$;

(iv) $\left\{\lambda_{n}\right\} \subset\left(0, \frac{2}{L}\right)$ for each $i \geq 1$, where $L=\|G\|^{2}$,

then the sequence $\left\{w_{n}\right\}$ converges strongly to $w^{*}=P_{\Gamma_{2}} f\left(w^{*}\right)$, which is a solution of $(\operatorname{SEEP}(h$, $g, D))(4.5)$. 
Let $H_{1}$ and $H_{2}$ be two real Hilbert spaces, $A: H_{1} \rightarrow H_{2}$ be a linear and bounded operators, $h: H_{1} \rightarrow \mathbb{R}$ and $g: H_{2} \rightarrow \mathbb{R}$ be two proper convex and lower semicontinuous functions. The split optimization problem (SOP) is to find $x^{*} \in H_{1}, A x^{*} \in H_{2}$ such that

$$
h\left(x^{*}\right)=\min _{x \in H_{1}} h_{i}(x) \text { and } g\left(A x^{*}\right)=\min _{z \in H_{2}} g(z) .
$$

Denote $U=\partial h$ and $K=\partial g$, then the (SOP) (4.9) is equivalent to the following split variational inclusion problem (SVIP): to find $x^{*} \in H_{1}$ such that

$$
0 \in U\left(x^{*}\right), \quad 0 \in K\left(A x^{*}\right) .
$$

In Theorem 4.1 taking $H_{3}=H_{2}, B=I$ (the identity mapping on $H_{2}$ ) and

$$
\tilde{G}=[A-I], \quad \tilde{G}^{*}=\left[\begin{array}{c}
A^{*} \\
-I
\end{array}\right], \quad \tilde{G}^{*} \tilde{G}=\left[\begin{array}{cc}
A^{*} A & -A^{*} \\
-A & I
\end{array}\right],
$$

then from Theorem 4.1 we have the following.

Corollary 4.5 Let $H_{1}, H_{2}, A, I, \tilde{G}, \tilde{G}^{*}, U, K$, be the same as above. Let $J_{\mu}^{(U, K)}$, $f$ be the same as in Theorem 4.1. For any given $w_{0}=\left(x_{0}, y_{0}\right) \in H_{1} \times H_{2}$, let $\left\{w_{n}=\left(x_{n}, y_{n}\right)\right\}$ be the sequence defined by

$$
\left\{\begin{array}{l}
x_{n+1}=\alpha_{n} x_{n}+\beta_{n} f_{1}\left(x_{n}\right)+\gamma_{n} J_{\mu}^{U}\left(x_{n}-\lambda_{n} A^{*}\left(A x_{n}-y_{n}\right)\right), \\
y_{n+1}=\alpha_{n} y_{n}+\beta_{n} f_{2}\left(y_{n}\right)+\gamma_{n} J_{\mu}^{K}\left(y_{n}+\lambda_{n}\left(A x_{n}-y_{n}\right)\right),
\end{array}\right.
$$

or its equivalent form:

$$
w_{n+1}=\alpha_{n} w_{n}+\beta_{n} f\left(w_{n}\right)+\gamma_{n}\left(J_{\mu}^{(U, K)}\left(I-\lambda_{n} \tilde{G}^{*} \tilde{G}\right) w_{n}\right), \quad n \geq 0,
$$

If $\Gamma_{3}:=\left\{x^{*} \in U^{-1}(0) \cap A^{-1} K^{-1}(0)\right\}$, the solution set of (SOP) (4.9) is nonempty, and the following conditions are satisfied:

(i) $\alpha_{n}+\beta_{n}+\gamma_{n}=1$, for each $n \geq 0$;

(ii) $\lim _{n \rightarrow \infty} \beta_{n}=0$, and $\sum_{n=0}^{\infty} \beta_{n}=\infty$;

(iii) $\liminf _{n \rightarrow \infty} \alpha_{n} \gamma_{n}>0$;

(iv) $\left\{\lambda_{n}\right\} \subset\left(0, \frac{2}{L}\right)$, where $L=\|\tilde{G}\|^{2}$,

then the sequence $\left\{w_{n}\right\}$ converges strongly to $w^{*}=P_{\Gamma_{3}} f\left(w^{*}\right)$, which is a solution of $(S O P)$ (4.9).

Competing interests

The authors declare that they have no competing interests.

Authors' contributions

All authors read and approved the final manuscript.

\section{Acknowledgements}

The authors would like to express their thanks to the editors and the referees for their helpful suggestion and advices.

This work was supported by the National Natural Science Foundation of China (Grant No. 11361070). 


\section{References}

1. Censor, Y, Elfving, T: A multiprojection algorithm using Bregman projections in a product space. Numer. Algorithms 8 221-239 (1994)

2. Byrne, C: Iterative oblique projection onto convex subsets and the split feasibility problem. Inverse Probl. 18, 441-453 (2002)

3. Censor, Y, Bortfeld, T, Martin, N, Trofimov, A: A unified approach for inversion problem in intensity-modulated radiation therapy. Phys. Med. Biol. 51, 2353-2365 (2006)

4. Censor, Y, Elfving, T, Kopf, N, Bortfeld, T: The multiple-sets split feasibility problem and its applications. Inverse Probl. 21, 2071-2084 (2005)

5. Censor, Y, Motova, A, Segal, A: Perturbed projections ans subgradient projections for the multiple-sets split feasibility problem. J. Math. Anal. Appl. 327, 1244-1256 (2007)

6. Xu, HK: A variable Krasnosel'skii-Mann algorithm and the multiple-sets split feasibility problem. Inverse Probl. 22, 2021-2034 (2006)

7. Yang, Q: The relaxed CQ algorithm for solving the split feasibility problem. Inverse Probl. 20, 1261-1266 (2004)

8. Zhao, J, Yang, Q: Several solution methods for the split feasibility problem. Inverse Probl. 21, 1791-1799 (2005)

9. Chang, SS, Cho, YJ, Kim, JK, Zhang, WB, Yang, L: Multiple-set split feasibility problems for asymptotically strict pseudocontractions. Abstr. Appl. Anal. 2012, Article ID 491760 (2012). doi:10:1155/2012/491760

10. Chang, SS, Wang, L, Tang, YK, Yang, L: The split common fixed point problem for total asymptotically strictly pseudocontractive mappings. J. Appl. Math. 2012, Article ID 385638 (2012). doi:10.1155/2012.385638

11. Moudafi, A: A relaxed alternating CQ algorithm for convex feasibility problems. Nonlinear Anal. 79, 117-121 (2013)

12. Moudafi, A, Al-Shemas, E: Simultaneous iterative methods for split equality problem. Trans. Math. Program. Appl. 1, 1-11 (2013)

13. Eslamian, M, Latif, A: General split feasibility problems in Hilbert spaces. Abstr. Appl. Anal. 2013, Article ID 805104 (2013)

14. Chen, RD, Wang, J, Zhang, HW: General split equality problems in Hilbert spaces. Fixed Point Theory Appl. 2014, Article ID 35 (2014)

15. Chuang, C-S: Strong convergence theorems for the split variational inclusion problem in Hilbert spaces. Fixed Point Theory Appl. 2013, Article ID 350 (2013)

16. Chang, S-S, Wang, L: Strong convergence theorems for the general split variational inclusion problem in Hilbert spaces. Fixed Point Theory Appl. 2014, Article ID 171 (2014)

17. Ansari, QH, Rehan, A: Split feasibility and fixed point problems. In: Ansari, QH (ed.) Nonlinear Analysis: Approximation Theory, Optimization and Applications, pp. 282-322. Springer, New York (2014)

18. Blum, E, Oettli, W: From optimization and variational inequalities to equilibrium problems. Math. Stud. 63(1/4), 123-145 (1994)

19. Moudafi, A: Split monotone variational inclusions. J. Optim. Theory Appl. 150, 275-283 (2011)

20. Censor, Y, Segal, A: The split common fixed point problem for directed operators. J. Convex Anal. 16, 587-600 (2009)

21. Naraghirad, E: On an open question of Moudafi for convex feasibility problem in Hilbert spaces. Taiwan. J. Math. 18(2), $371-408(2014)$

22. Chang, SS, Joseph Lee, HW Chan, CK, Zhang, WB: A modified Halpern-type iterative algorithm for totally quasi-asymptotically nonexpansive mappings with applications. Appl. Math. Comput. 218, 6489-6497 (2012)

23. Chang, SS: On Chidume's open questions and approximate solutions for multi-valued strongly accretive mapping equations in Banach spaces. J. Math. Anal. Appl. 216, 94-111 (1997)

24. Maingé, P-E: Strong convergence of projected subgradient methods for nonsmooth and nonstrictly convex minimization. Set-Valued Anal. 16(7-8), 899-912 (2008)

25. Goebel, K, Kirk, WA: Topics in Metric Fixed Point Theory, vol. 28. Cambridge University Press, Cambridge (1990)

10.1186/1687-1812-2014-215

Cite this article as: Chang et al.: Moudafi's open question and simultaneous iterative algorithm for general split equality variational inclusion problems and general split equality optimization problems. Fixed Point Theory and Applications 2014, 2014:215

\section{Submit your manuscript to a SpringerOpen ${ }^{\circ}$ journal and benefit from:}

- Convenient online submission

- Rigorous peer review

- Immediate publication on acceptance

- Open access: articles freely available online

- High visibility within the field

- Retaining the copyright to your article 